\title{
GLOBAL MINDSET E COMPORTAMENTO DA LIDERANÇA
}

\section{RESUMO}

Global Mindset, conjunto de atributos e características que auxiliam o líder global a influenciar indivíduos, grupos e organizações que estão em qualquer parte do mundo tem sido descrito como um fator crítico para o desenvolvimento organizacional.

Para identificar os fatores que afetam o global mindset de líderes globais e o seu efeito no seu comportamento, neste trabalho com base no modelo de Konyu-Fogel(2011) foi aplicado um questionario on line para uma amostra de 163 líderes de que atuam em atividades com escopo internacional de organizações multinacionais ou nacionais, em vinte e duas indústrias na África, Asia, América do Sul e do Norte, Europa e no Oriente Médio.

Os resultados do trabalho evidenciaram que o global mindset é responsável por até 39,1\% de variabilidade no comportamento da liderança, confirmando estudos prévios de que líderes globais efetivos usam comportamentos que mostram um entendimento das diferenças entre culturas e países em resposta ao ambiente.

Especificamente no que diz respeito a fatores demográficos como idade, gênero, número de línguas estrangeiras faladas, número de países trabalhados e a criação em família bilíngüe/multiétnica), os resultados demonstraram que eles não afetam significantemente o global mindset., diferente de fatores como, local de trabalho e posição. Por fim, na avaliação do efeito dos fatores organizacionais sobre o global mindset, identificamos o impacto significativo da linha de produto(negócio), e o percentual de receita do exterior no global mindset dos líderes.

Palavras-chave: liderança global, Global Mindset, comportamento da liderança

\section{GLOBAL MINDSET AND LEADERSHIP BEHAVIOR}

\section{ABSTRACT}

Global Mindset, set of attributes and characteristics that help global leadership to influence individuals, groups and organizations that are anywhere in the world has been described as a critical factor for organizational development.

In order to identify factors that affect the Global Mindset of leaders, an on-line survey based on Konyu-Fogel (2011) model was applied to a sample of 163 leaders that work in positions that require international activities in organizations in twenty-two product lines (industries) in Africa, Asia,, South and North America, Europe and Middle East.

We conclude that Global Mindset is responsible for 39,1\% of variability on leadership behavior, confirming previous studies which identified that global leaders with high Global Mindset are more likely to exhibit leadership behaviors which present understanding on differences across cultures and countries in response to the global environment.

Specifically related with demographic factors such as age, gender, number of foreign languages spoken, number of countries worked in and raised/lived in bilíngüe/multiethnic family, the results showed that they do not affect Global Mindset score, different from the factors such as local of employment and posicion.

Finnaly, on assessment of organizational factors effects on Global Mindset, we identified a weak relationship under total number of employees, percentage of employees working overseas, location/country of headquarter's and Global Mindset. On the other hand, product line and percentage of revenue from foreign operations significantly affect business leaders Global Mindset.

Keywords: Global Mindset, global leadership, leadership behavior 


\section{RESUMEN}

\section{GLOBAL MINDSET Y EL COMPORTAMIENTO DE LIDERAZGO}

Global Mindset, atributos y características de ajuste que ayudan liderazgo global para influir en las personas, grupos y organizaciones que están en cualquier parte del mundo ha sido descrito como un factor crítico para el desarrollo de la organización.

Para identificar los factores que afectan o Global Mindset de líderes y su efecto sobre su comportamiento, una encuesta en línea basado en Konyu - Fogel (2011) modelo se aplicó a una muestra de 163 líderes que trabajan en puestos que requieren las actividades internacionales en las organizaciones en veintidós producto líneas (industrias) en África, Asia, América del Sur y del Norte, Europa y Oriente Medio.

Los resultados de estúdio mostraram que el Global Mindset es responsable de hasta el 39,1\% de la variabilidad en el comportamiento de liderazgo, lo que confirma estudios previos que identificaron que los líderes mundiales con alta Global Mindset son más propensos a mostrar comportamientos que muestran una comprensión de las diferencias entre las culturas y los países en respuesta al ambiente.

Específicamente con respecto a los factores demográficos (edad, género y número de idiomas hablados, número de países trabajados y a creación en família bilíngüe/multiétnica)), los resultados demostraron que no afectan significativamente el Global Mindset score, diferente de los factores como, local de trabajo y posición.

Por ultimo, al evaluar el efecto de los factores organizativos sobre el Global Mindset, hemos identificado el impacto significativo de la línea de productos (negocio) la ubicación de la matriz y el porcentaje de los ingresos en el extranjero sobrel el Global Mindset.

Palabras clave: Global Mindset, liderazgo global, comportamiento de liderazgo

\footnotetext{
${ }^{1}$ Pontificia Universidade Catolica - PUC, Rio de Janeiro, Brasil

E-mail: vania.1.neves@gsk.com

${ }^{2}$ Pontificia Universidade Catolica - PUC, Rio de Janeiro, Brasil

E-mail: patomei@iag.puc-rio.br
} 


\section{INTRODUÇÃO}

A economia globalizada, com requisitos diferentes dos modelos anteriores de economia internacional, multinacional e comercial, cria novas realidades de negócios que devem ser reconhecidas pelas organizações e seus gestores. Os imperativos da globalização se aplicam à maioria das indústrias, impactando as organizações em todos os níveis hierárquicos da cadeia gerencial (Jeannet, 2000).

As últimas décadas foram marcadas por transformações profundas em todo o mundo estimuladas pela globalização. A magnitude destas mudanças teve impactos econômicos, sociais e culturais em escala mundial. Entre 1990 e 2003, o número de organizações multinacionais no mundo cresceu de 3000 para 63.000(Gabel e Bruner, 2003). Em 2012, havia mais de 100.000 multinacionais no mundo e 900.000 afiliadas, com patrimônio de \$57 trilhões. Em pesquisa recente com 500 executivos de 100 organizações, o Economist Intelligence Unit reportou que $75 \%$ destas organizações planejavam competir em mercados externos. (Unit,2010)

Em pesquisa recente, hum mil trezentos e quarenta e quatro $C E O$ 's de 68 paises reportaram a PriceWaterCoopers (2014) que tinham o desafio de liderar seus negócios dentro do cenário instável da economia global atual e planejavam buscar novas oportunidades de crescimento dentro e fora dos países onde já atuavam. Para responder às tendências globais atuais, ressaltaram a necessidade de profissionais que gerenciem um time global multigeracional $e$ multicultural, com criatividade sistemática, esejam capazs de satisfazer as necessidades dos clientes que estão mais exigentes do que nunca. Como resultado, $63 \%$ dos CEO's reportaram preocupação com a disponibilidade de profissionais com o perfil de liderança necessários para o desafio atual.

O World Economic Forum (2015) publicou pesquisa realizada com hum mil setecentos e sessenta e sete executivos de todos os continentes, que $86 \%$ desta amostra respondeu que atualmente há uma crise global de liderança no mundo, justificada pela comunidade internacional ter falhado fortemente em resolver as crises globais nos anos recentes.

Uma das consequências da globalização é que as organizações necessitam de líderes que tenham uma habilidade de integrar diferentes pontos de vistas para atingir produção em escala mundial, marketing e sistema de entrega de valor global (Jeannet, 2000). O desafio global não é somente preparar a força de trabalho futura, mas fazê-lo de forma que se preserve o papel da escolha fundamental focando as mentes na visão ampliada (Global Risk Report, 2014).

Global Mindset, conjunto de atributos e características que auxiliam o líder global a influenciar indivíduos, grupos e organizações que estão em qualquer parte do mundo (Javidan e Bowen, 2013b), tem sido descrito como um fator crítico para o desenvolvimento e sucesso da liderança global (Kedia e Mukherji, 1999;Black e Gregersen, 2000; Jeannet, 2000; Oddou et.al., 2000; Levy et al., 2007; Cohen, 2010; Javidan e Bowen, 2013b;2013a; Javidan e Walker, 2013).Estratégias de negócios bem sucedidas devem começar com a transformação no mindset cognitivo global da liderança(Aggarwal, 2011).

Líderes com global mindset tem uma visão global mais ampliada, uma orientação global para o negócio e são adaptáveis a cultura e ambiente local (Story e Barbuto, 2011). Líderes globais efetivos usam comportamentos que mostram um entendimento das diferenças entres culturas e países em resposta a ambientes globais e execução de tarefas complexas(Goldsmith et al., 2003).

De acordo com pesquisa realizada com cento e cinquenta e oito respondentes de multinacionais que atuavam em posições de liderança global, o nível do comportamento da liderança que é crítico para a performance da organização pode aumentar quando global mindset é maior(Konyu-Fogel,2011). Esta análise, focada prioritariamente em liderenças norte americanas, precisa ser expandida com o exame do efeito do global mindset em amostras maiores e diferentes indústrias/países, provendo mais insumos para as organizações atuarem no desenvolvimento de seus líderes globais.

Este é o objetivo geral deste trabalho, ampliar o estudo de Konyu-Fogel(2011) e explorar o efeito do global mindset no comportamento da liderança em uma amostra de líderes globais, de organizações multinacionais e nacionais que atuam na África, Asia, América do Sul, América do Norte, Europa e Oriente Médio.

\section{REFERENCIAL TEÓRICO 2.1 LIDERANÇA GLOBAL}

A globalização promove o crescimento dos países (Dreher, 2006). Contudo, a aceleração da globalização tem criado estado de caos de mudança enquanto as organizações lutam para se adaptar a novos paradigmas de liderança, para os quais a abordagem de tentativa e erro não é mais efetiva (Robinson e Harvey, 2008), requerendo que líderes possuam não somente as habilidades de liderança como também um conjunto de habilidades para demonstrar eficácia no mercado global, gerenciando a complexidade entre culturas com empregados, clientes, fornecedores e competidores (Blaess, et.al., 2012; Dorfman et al., 2012).

Em 1998, na pesquisa Global Leader of Future Network Forum (Goldsmith et al., 2003), duzentos e dois líderes responderam um questionário de 82 questões, no qual citaram exemplos de liderança global efetiva que foram hierarquizados segundo ordem de importancia importância e agrupados quanto ao impacto de seu trabalho (ex. Bill Gates e Wiston 
Churchil), de suas posições e mandatos globais (ex.o Papa e Koffi Annan das Nações Unidas) e de seu exemplo e legado (ex. Gandhi e Nelson Mandela).

A revista Fortune (2014) em sua lista dos 50 maiores líderes do mundo que inspiram e energizam seus seguidores e constroem um mundo melhor incluiu o Papa Francisco, Bill Clinton, Angela Merckel e Dalai Lama, e a revista Forbes(2014) cita Vladmir Putin, Barack Obama, Xi Jinping e Mark Zuckerberg.

Todos estes indivíduos apresentam características de liderança global efetiva, cujo impacto e seguidores atravessam fronteiras, e que segundo Goldsmith et al., (2003) e Gundling et.al. (2011) demonstram autoconfiança, criam e comunicam sua visão, consistentemente tratam pessoas com respeito e dignidade, tem consciência cultural e são frequentemente capturados na imaginação de uma ampla audiência devido a confluência de suas visões e relevância para o contexto mundial (Mendenhall et al.,2013).

Não há consenso sobre a definição de liderança global no que diz respeito a rigor, precisão e escopo, variando a abordagem em relação às fronteiras da designação de líder global. Mendenhall et al. (2012) analisaram tres dimensões críticas para endereçar os elementos contextuais, relacional e temporal-espacial do constructo: (a) complexidade da operação e residência (b) fronteiras para dentro e fora da organização, (c) grau de colocação geográfica e enfatizarm que a liderança global está relacionada a um processo que influencia outros a adotar um visão compartilhada em um contexto caracterizado por níveis significativos de complexidade, fluxo e presença.

Beechler e Javidan (2007) definem liderança global como o processo de influenciar indivíduos, grupos e organização (dentro e fora das fronteiras globais da organização) representando sistemas culturais, políticos e institucionais diversos para contribuir com o cumprimento das metas da organizaçãoç e Morrison (2000) diferenciou as características que são gerais, aplicáveis universalmente e de outras que são específicas que variam de acordo com as condições locais e identificou que modelos de liderança doméstica de uma parte do mundo geralmente não funcionam mundialmente devido às diferenças culturais.

Inúmeras pesquisas têm sido realizadas com objetivo de compreender a forma de atuação de uma liderança global que opera em países diferentes. O projeto Global Leadership and Organizational Behavior Effectiveness(GLOBE) é considerado por muitos autores um dos estudos mais ambiciosos sobre o tema (Morrison, 2000). Ele envolveu cento e sessenta cientistas sociais e estudiosos de gestão de 62 culturas, mais de dezessete mil gerentes de mais de 900 organizações e analisou os efeitos da cultura sobre a efetividade demonstrando que enquanto países diferentes possuem visões divergentes em muitos aspectos, há questões universais referentes a atuação da liderança global (Javidan et al., 2006; Dorfman et al., 2012).

Neste projeto são definidas seis dimensões para liderança global: carismática, autêntica, participativa, orientada para pessoas, independente e individualista.e se identifica que os valores culturais influenciam o comportamento da liderança,isto é, os atributos da liderança tendem a ser alinhados com os valores culturais da sociedade e que os líderes que se comportam de acordo com as expectativas da sociedade são mais efetivos. (Dorfman et al., 2012).

Com a globalização há uma mudança de fornecimento para mercados externos de uma base doméstica para estabelecer subsidiárias em vários países, através de fusões e aquisições de organizações estrangeiras ou joint ventures. (House et. al., 2001). No processo de mudança e desenvolvimento de uma organização internacional, a adequação ou conflito entre a sua cultura e a cultura nacional é um desafio para a liderança(Bowen e Inkpen, 2009) e requer um tipo especial de líder capaz de integrar empregados de diferentes culturas (House et.al, 2002;Goldsmith et al., 2003; Gundling et.al., 2011).

Negócios internacionais estão sujeitos a um amplo escopo de interpretações que podem fazer o entendimento assim como a cooperação impossível (Earley e Mosakowski, 2004). Em um cenário onde cruzar fronteiras é um rotina, a inteligência cultural - a habilidade do indívíduo para interpretar gestos ambíguos e não familiares de alguém da forma igual a de seus compatriotas - é um habilidade importante para a liderança global(Earley e Mosakowski, 2004; Triandis, 2006).

Diversos pesquisadores contribuiram com suas visões para a literatura sobre competências da liderança global - qualidades, habilidades, valores e tratos pessoais que são essenciais para desempenhar atividades específicas que são requeridas para os líderes operarem entre fronteiras nacionais em ambientes globais complexos(Beechler e Javidan, 2007; Gundling et al. ,2011; Tubbs e Schulz, 2006) e comportamento da liderança global - conjunto de atitudes que valorizam compartilhar informação, e experiência entre fronteiras nacionais, funcionais e de negócios, balanceando a competição de prioridades funcionais, de negócio e de países que emergem no processo de globalização(Pucik e Saba, 1998).

Para Morley e Cerdin (2010) as competências para liderança global estão relacionadas à capacidade de desempenho efetivo em situação entre-culturas e para ser bem sucedido no ambiente internacional operacional, os líderes globais necessitam ser capazes de sentir e interpretar as mudanças do ambiente dinâmico e complexo, integrar múltiplas estratégias e capitalizar no potencial competitivo vantagens inerentes em seu posicionamento de mercado mundial (Bartlett e Beamish, 2014).

Brake(1997) criou o modelo Global Leadership Triad (Tríade de Liderança Global), com 
três características de liderança:gerenciamento de relaciomento, perspicácia sobre o negócio e efetividade pessoal.Este modelo foi complementado por Goldsmith et al. (2003) com componentes como pensar globalmente e apreciar a diversidade cultural.

Uma competência importante do líder global é a inteligência cultural (Earley e Mosakowski, 2004) que favorece o reconhecimento e o entendimento das várias dimensões culturais e comportamento culturalmente aceitável entre culturas (Trompennars e Hampden-Turner, 1998; Dorfman et al., 2012). A tomada de decisão em ambiente complexos e a habilidade para ler as nuances culturais e adaptar o estilo de liderança apropriadamente foi reportado como chave para o sucesso da liderança global (Javidan e Bowen, 2013).

Segundo Konyu-Fogel(2011), apesar da literatura indicar uma lista interminável de competências para liderança global, não foi identificada uma hierarquia comum de competências ou comportamentos para os líderes globais. Segundo a pesquisadora a listagem de forma prescritiva das competências globais pode desprezar a complexidade do fenômeno da liderança e sugeriu para melhor entendimento do conceito que diversos fatores do comportamento de liderança global fossem explorados através de estudos empíricos que ajudassem a entender a relação entre global mindset e comportamento da liderança.

\subsection{MINDSET LOCAL E GLOBAL}

Mindset é o termo em inglês que significa "mentalidade", a maneira individual de pensar e de julgar. $\mathrm{O}$ mindset gerencial está relacionado à capacidade cognitiva para tomada de decisão, com a qual o líder se posiciona diante de uma situação (Liedtka, 1991).

Perlmutter (1969) foi o precursor na literatura a propor uma tipologia para organizações multinacionais, baseada em mindsets gerenciais. Seu estudo desenvolvido ao longo de uma década (Heenan e Perlmutter,1979) identificou três estados da mente ou atitudes para tomada de decisão de produtos, funções e geografia: etnocêntrico (mindset doméstico, orientado para o país natal), policêntrico (mindset orientado para o país da matriz) e geocêntrico (global mindset, orientado para o mundo).
Segundo Jeannet (2000) a mentalidade doméstica se refere a indivíduos dos quais todos os pontos de referência são provenientes de uma cultura única que geralmente tem dificuldade em absorver idéias de outras partes do mundo e tendem a não terem o que ele chamou de curiosidade internacional, que é aprender sobre outras partes do mundo. Para o autor, indivíduos que tem mentalidade global são capazes de entender um negócio, um setor da indústria, um segmento particular de mercado sem se restrigirem a um país ou região.

Líderes locais são importantes para a organização, porém, não possuem todas habilidades para a organização competir em escala global(Levy et al., 2007). Geocêntrico global mindset demanda líderes globais que inspirem um grupo de pessoas a desejarem perseguir uma visão positiva de uma forma organizada e eficaz, enquanto promovem crescimento individual e coletivo em um contexto caracterizado por níveis significativos de complexidade, fluxo e presença (Mendenhall et. al., 2012). Estes líderes globais são caracterizados como indivíduos que geram mudanças positivas e significativas na organização, construindo comunidades através do desenvolvimento de confiança e ajuste de estruturas organizacionais e processos em um contexto envolvendo múltiplos stakeholders entre fronteiras e múltiplas culturas sobre condições de complexidade cultural, geográfica e temporal (Mendenhall et al., 2013). Eles têm uma perspectiva global do negócio; aprendem sobre as diferentes perspectivas de culturas, gostos, tendências, tecnologias para conduzir o negócio; trabalham com pessoas de várias culturas simultaneamente; se adaptam e interagem com colegas estrangeiros sem utilizar sua autoridade formal (Adler e Bartlomew ,1992).

Global mindset é a essência da liderança do século XXI e requer pessoas que possam pensar e entender globalmente desafios e oportunidades (Javidan e Walker, 2013).

A Tabela 1, apresenta algumas das principais definições disponíves na literatura para global mindset, demonstrando que apesar da diversidade de visões dos pesquisadores, os conceitos englobam qualidades ou filosofias pessoais, conhecimento e habilidades, comportamentos ou uma combinação destes(Ananthram e Nankervis, 2014). 
Tabela 1: Definições e Conceitos de Global Mindset

\begin{tabular}{|c|c|}
\hline Autores & Descrição \\
\hline $\begin{array}{l}\text { Rhinesmith (1996); } \\
\text { Jeanett (2000) }\end{array}$ & $\begin{array}{l}\text { Estado da mente capaz de entender um negócio, uma indústria ou um mercado particular } \\
\text { em base mundial. }\end{array}$ \\
\hline Calori et.al.(1994) & $\begin{array}{l}\text { Estrutura cognitiva ou map mental que permite o } C E O \text { compreender a complexidade de } \\
\text { um ambiente mundial da empresa. }\end{array}$ \\
\hline Kefalas (1998) & $\begin{array}{l}\text { Preposição para ver o mundo de formaparticular que estabelece fronteiras e explicações } \\
\text { do porque as coisas são do jeito que são e guia comportamentos. }\end{array}$ \\
\hline $\begin{array}{l}\text { Arora } \text { et al., (2004); } \\
\text { Taylor et al.,(2008); } \\
\text { Lovvorn e Chen } \\
(2011)\end{array}$ & Ligado a inteligência emocional e cultural com o foco na apreciação à diversidade cultural \\
\hline Levy et.al. (2007) & $\begin{array}{l}\text { Estrutura cognitiva altamente complexa caracterizda pela abertura para culturas multiplas } \\
\text { e realidades estratégicas em nível global e local, além da habilidade cognitiva para mediar } \\
\text { e integrar através de multiplicidade }\end{array}$ \\
\hline $\begin{array}{l}\text { Gupta e Govindarajan } \\
(2002)\end{array}$ & $\begin{array}{l}\text { Estrutura de conhecimento combina uma abertura para a consciência da diversidade entre } \\
\text { culturas e mercados com a propensão e habilidade de sintetizar através da diversidade. }\end{array}$ \\
\hline $\begin{array}{l}\text { Nummela et.al., } \\
(2004)\end{array}$ & $\begin{array}{l}\text { Atitude positiva na direção de negócios internationais e habilidadede de se ajustar a } \\
\text { diferentes costumes e culturas. }\end{array}$ \\
\hline Levy et al. (2007) & Inclui um domínio cultural e estratégico. \\
\hline Maak \& Pless (2009) & $\begin{array}{l}\text { Adiciona a perspectiva de "cosmopolitanismo", o qual se traduz em qualidades } \\
\text { específicas de liderança global. }\end{array}$ \\
\hline $\begin{array}{l}\text { Ananthram et.al. } \\
(2010)\end{array}$ & $\begin{array}{l}\text { Habilidade e desejo dos líderes para pensar, agir e transcender fronteiras e metas, valores } \\
\text { e competências em uma escala global. }\end{array}$ \\
\hline $\begin{array}{l}\text { Rogers e Blonski } \\
(2010)\end{array}$ & $\begin{array}{l}\text { Capacidade de engajar em um processo cognitive sintetizado e sem fronteiras que } \\
\text { identifica oportunidade e inovação em complexidade. }\end{array}$ \\
\hline Konyu-Fogel(2011) & $\begin{array}{l}\text { Inteligência intelectual(a habilidade de diferenciar e integrar informação) e a inteligência } \\
\text { cultural(estrutura cognitiva para organização e entendimento cultural sugerido para } \\
\text { adaptar e decretar comportamento em ambientes de diferentes culturas ) }\end{array}$ \\
\hline Javidan et al. (2013) & $\begin{array}{l}\text { Conjunto de atributos e características que ajudam aos líderes globais a influenciar melhor } \\
\text { indivíduos, grupos e organizações diferentes deles. Apresenta três dimensoes principais: } \\
\text { capital intelectual, capital psicológico e capital social. }\end{array}$ \\
\hline Lane et al.( 2004) & $\begin{array}{l}\text { Habilidade de desenvolver e interpretar critérios para desempenho pessoal e do negócio } \\
\text { que são independentes das premissas de país, cultura ou contextos únicos. }\end{array}$ \\
\hline $\begin{array}{l}\text { Story et.al } \\
(2014)\end{array}$ & $\begin{array}{l}\text { Combinação de altos níves de inteligência cultural(congnitiva, motivacional e } \\
\text { inteligência cultural meta-cognitiva) e orientação ao negócio global, além da habilidade } \\
\text { de se adaptar ao ambiente e cultural local. }\end{array}$ \\
\hline
\end{tabular}

Fonte: Diferentes conceitos de global mindset(Ananthram e Nankervis, 2014), adaptado pelos autores. 
Global Mindset e Comportamento da Liderança

\subsection{DESENVOLVIMENTO E MENSURAÇÃO DO GLOBAL MINDSET}

Não há consenso entre os pesquisadores sobre como o global mindset é adquirido. Há pesquisadores que sugerem que historicamente está dentro dos indivíduos de forma nativa, e outros que afirmarm que este se desenvolve com experiência global contínua ao longo do tempo(Bouquet et.al. , 2009).

Beechler e Javidan (2007) colocam que embora algumas características do global mindset sejam consideradas genéticas, ele pode ser desenvolvido através de socialização, como um processo evolutivo construído sobre mecanismos de feedback que expandem os esquemas mentais de um indivíduo(Lovvorn e Chen, 2011) proporcionando uma mudança na mentalidade gerencial (Fatehi e Ghadar, 2014).

Vários métodos e ferramentas gerenciais são apresentadas na literatura para o desenvolvimento do global mindset como: experiências com viagens e expatriação (Javidan et al.,2006), participação em projetos e times globais além das fronteiras, networking, reuniões em diversas localidades, educação formal de cultura e idiomas (Beechler e Javidan, 2007), planejamento de carreira, responsabilidade global(Gupta et al., 2008), programas de treinamentos globais, experiências de trabalho com times multiculturais (Miriam et al., 2013), programas para desenvolvimento da inteligência cultural (Earley e Mosakowski, 2004), composição de time executivo multicultural, designações internacionais, job rotation através de vários negócios e diferentes designações em vários países(Paul, 2000).

São inúmeros os constructos para a mensuração do global mindset do indívíduo,dos quais destacamos: técnicas de mapeamento cognitivo para investigar o conhecimento complexo dos CEO's (Calori et al. ,1994), a avaliação da experiência internacional de times gerenciais (Sambharya,1996), medidas que associam comportamentos, habilidades e atitudes individuais. (Murtha et al., 1998; Calof e Beamish ,1994; Jeannet,2000) avaliações de práticas gerenciais (Gupta et al.,2008), a associação da inteligência intelectual com a inteligência cultural (Konyu-Fogel,2011), índice cognitivo de internacionalização da empresa para descobrir a discrepância ou mostrar o alinhamento entre a realidade do mercado e as atitudes gerenciais(Fatehi e Ghadar, 2014), o Thunderbird Global Mindset Inventory (TGMI) com 76 questões que medem o capital intelectual global, capital psicológico global e capital social global (Javidan e Bowen, 2013), o Global Competency Inventory (GCI) desenvolvido pelo The Kozai Group para autoavaliação em dezesseis diferentes áreas que são relacionadas com adaptação a pessoas e lugares(Mendenhall et. al., 2008), o Global Leader of the Future Inventory (GLFI) criado por
Greenberg et.al.(1999), com o objetivo de construir conhecimento sobre o futuro do líder executivo com um escopo global e validado por Goldsmith et al.(2003). Ranker et.al. (2015) definiram global mindset como um passo fora da base cultural do indivíduo para entender que não há forma universalmente correta para fazer coisas. Para os autores, desenvolver um global mindset significa aceitar que nossos valores e formas de fazer negócio não têm o mesmo significado ou talvez funcionem para nossos pares em outras culturas.

2.4 EFEITO DO GLOBAL MINDSET NO $\begin{array}{llll}\text { COMPORTAMENTO } & \text { DA LIDERANÇA } \\ \text { SEGUNDO MODELO } & \text { CONCEITUAL DE } \\ \text { KONYU-FOGEL(2011). } & & & \end{array}$

Konyu-Fogel(2011) conduziu uma pesquisa com cento e trinta e oito líderes globais que trabalhavam em organizações da lista Fortune a partir de três constructos: fatores demográficos e organizacionais, global mindset e comportamento da liderança. O global mindset foi operacionalizado com o constructo de (1) inteligência intelectual (habilidade de diferenciar e integrar informação) composto de diferenciação, integração, gerenciamento de incerteza, reconhecimento de padrão e pensamento global e (2) inteligência cultural (estrutura cognitiva para organização e entendimento cultural sugerido para adaptar e decretar comportamento de liderança em um determinado contexto) definido como abertura para aprendizado, sensibilidade emocional, comportamento flexível e entendimento entre culturas. O comportamento da liderança se refere as ações de planejamento, coordenação, liderança, comunicação, colaboração e motivação de pessoas.

No estudo de Knoyu-Fogel(2011), foi encontrada significância do global mindset com quatro fatores demográficos, sugerindo uma direta e positiva relação de líderes com global mindset e o número de países onde já trabalhou ou operou com, números de línguas estrangeiras faladas, criação em família bilingue/multiétnica e localidade/região do empregado. Também foi identificada significância do global mindset com dois fatores organizacionais, sugerindo uma direta e positiva relação de líderes com global mindset e o percentual de empregados trabalhando no exterior, percentual da receita oriundo de operação no exterior, e, confirmada a significância do global mindset sobre o comportamento da liderança.

$$
\text { Konyu-Fogel(2011) concluiu que o }
$$
desenvolvimento do global mindset é provável de ser influenciado por múltiplos fatores compreendidos de experiências pessoais, educacionais e profissionais e que o envolvimento da família, educação, governo e organização global são importantes no desenvolvimento do global mindset e habilidades de liderança global. 


\section{METODOLOGIA}

Este estudo caracterizou-se pelo método quantitativo, com questionário estruturado que gerou dados estatísticos, obtidos de uma amostra aleatória. $\mathrm{O}$ universo definido para pesquisa foi de líderes de organizações multinacionais ou nacionais que atuem em posições que requeiram liderança de negócios, atividades ou times com escopo internacional ou global.

A amostra da pesquisa foi construída a partir da rede de contatos das pesquisadoras, que incluiu trezentos executivos que trabalham e vivem nos continentes África, Ásia, Europa, América do Norte e América do Sul, que foram convidados a participação da pesquisa.

Este grupo recebeu uma carta convite via email explicando o propósito do estudo, garantindo a confidencialidade das respostas junto a um link para acesso a um questionário on-line no período de outubro a dezembro de 2014. Após o encerramento da coleta de dados, a amostra final para análise, considerou cento e trinta e seis participantes que completaram a pesquisa, com dados utilizáveis que ocupam posições de Proprietário/Presidente, Chief Executive Officer (CEO), Chief Information Officer (CIO), Chief Operating Officer (COO), Vice-Presidente, Diretor, Executivo de Marketing, Recruitment Partner (Parceiro de Recrutamento), Consultor em Gerenciamento, Gerente Senior, Gerente de Programa/Projeto, Gerente, Engenheiro Senior e Educador/Especialista em Treinamento.

Estes participantes atuam em vinte duas linhas de produtos(indústrias): Serviços Financeiros e
Bancários, Indústria Química, Commodities, Consultoria, Engenharia, Entretenimento, Acessórios e Moda, Alimentos e Bebidas, Governo, Health Care (Cuidados com Saúde), Seguros, Tecnologia da Informação, Logística, Consultoria em Gerenciamento, Manufatura, Marketing, Serviços Profissionais, Pesquisa e Desenvolvimento, Serviços Administrativos, Telecomunicações, Treinamento/Educação, Infraestrutura de Transporte.

Neste estudo foi utilizado o instrumento de pesquisa de Konyu-Fogel (2011). A pesquisadora e o co-autor do instrumento foram contatados $\mathrm{e}$ autorizaram a utilização de seu questionário, criado para sua tese de doutorado. O instrumento foi originalmente desenvolvido e estatisticamente testado para significância e de acordo com Cronbach's alpha tests e o resultado da análise confirmatória, o instrumento demonstrou confiabilidade forte e alta consistência interna (Konyu-Fogel ,2011). Apesquisadora também foi contatada por e-mail e telefone para esclarecimento de dúvidas sobre $\mathrm{o}$ questionário e a análise estatística utilizada.

A primeira seção do questionário, é composta de 13 itens relativos aos fatores demográficos e organizacionais, usados para identificar quais fatores podem influenciar/aumentar o global mindset do líder. A segunda é composta de 18 itens para classificação das atitudes e percepções em relação ao global mindset em uma escala Likert de 1-5. O cálculo da escala completa do global mindset é obtido pela média dos valores de cada um dos 16 itens da Escala Global mindset apresentados na Tabela 2 a seguir:

Tabela 2 Escala Global Mindset de Konyu-Fogel (2011)

Inteligência Intelectual (9 items)
Diferenciação e Integração
Entende diferenças culturais/econômincas que impactam o negócio
Avalia confortavelmente riscos/oportunidades em um negócio global
Incorpora múltiplos pontos de vista de diferentes culturas
Gerenciamento de Incerteza
Trabalha bem com ambiguidade e situações não estruturadas
Pode lidar com decisões sob incerteza ou restrição de tempo
Reconhecimento de Padrão
Apresenta/analiza confiante questões complexas de negócio
Avalia negócio global e ambientes de países estrangeiros
Pensamento Global
Regularmente monitora notícias internacionais e eventos mundiais
Tenta aprender sobre as práticas de negócios de outros países
Inteligência Cultural (7 items)




\begin{abstract}
Abertura para aprendizado
Gosta de viajar/encontrar pessoas de outros países/culturas

Gosta de trabalhar com pessoas de países/culturas diferentes

Flexibilidade e Sensibilidade

Se comunica bem em diferentes culturas/países

Entende culturas e tradições de outros países

Entende que valores culturais influenciam práticas de negócios

Entendimento entre Culturas

Entende que diferenças culturais fornecem vantagens competitivas ao negócio Respeita as histórias/tradições/culturas de outros países
\end{abstract}

Fonte: Konyu-Fogel (2011). Adaptado pelos pesquisadores

A terceira seção do questionário é composta de 20 itens para classificação dos participantes quanto às suas percepções sobre as seis dimensões de
Comportamento da Liderança, em uma escala Likert de 1-5, apresentada na Tabela3

Tabela 3 Escala Comportamento da Liderança de Konyu-Fogel (2011)

\section{Planejamento(3 items) \\ Avalia oportunidades/desafios/riscos globais para o negócio Analisa fatores econônicos/culturais que impactam o negócio Solicita informação de negócios fora do país de origem}

\section{Coordenação(4 items)}

Avalia múltiplos pontos de vista para resolver questões de negócio Usa sistemas de produção/marketing externo ao país de origem Integra cadeia de fornecedores entre diferentes países Compartilha tomada de decisão com executivos de outros países

\section{Leading (4 items)}

Desenvolve valores organizacionais que representam outras culturas Delega autoridade e designa atividades entre culturas Seleciona/promove pessoas de um banco global de talentos Engaja-se em treinamento entre culturas e desenvolve subordinados

\section{Motivação (3 items)}

Respeita realizações de empregados de outra culturas Usa reconhecimentos para refletir valores dos empregados de outras culturas Encoraja engajamento entre culturas para atingir metas

\section{Comunicação (3 items)}

Busca visões de "stakeholders" (interessados) de diferentes países Responde a diferenças em práticas de negócios e culturas Participa de compartilhamento de informações gobais entre culturas

\section{Colaboração (3 items)}

Trabalha com intituições civis entre culturas

Usa redes entre culturas para alcançar os objetivos do negócio

Desenvolve alianças/parcerias/"venture" entre culturas 
Foram usadas as seguintes hipóteses de pesquisa: $\mathrm{H}_{1}$ : Os fatores demográficos influenciam o global mindset dos líderes de negócios.

$\mathrm{H}_{2}$ : Os fatores organizacionais influenciam o global mindset dos líderes de negócios.

H3: O global mindset dos líderes de negócio afeta o comportamento da liderança que é critico para o desempenho organizacional.

Para testar as hipóteses definidas, foram usadas as variáveis dependentes e independentes. Para testar as hipóteses 1 e 2, nas quais os fatores demográficos e organizacionais hipoteticamente influenciam o global mindset, foram usadas 13 variáveis independentes (7 demográficas e 6 organizacionais); a variável dependente é a escala completa do Global Mindset.Para testar as hipótese 3, na qual o global mindset hipoteticamente afeta o comportamento da liderança, a variável independente é a escala completa do Global mindset; a variável dependente é a escala completa do Comportamento da Liderança conforme resumido na Tabela 4 a seguir:

Tabela 4: Hipoteses e Variáveis Dependentes e Independentes

\begin{tabular}{|c|c|c|}
\hline Hipóteses & Variáveis Independentes & Variável Dependentes \\
\hline Hipótese 1 & $\begin{array}{l}\text { Fatores Demográficos } \\
\text { Idade } \\
\text { Gênero } \\
\text { Posição } \\
\text { № de países trabalhou } \\
\text { em/operou com } \\
\text { № línguas estrangeiras faladas } \\
\text { Criado por uma família } \\
\text { bilingue/multiétnica } \\
\text { Local de trabalho }\end{array}$ & Escala Completa Global Mindset \\
\hline Hipótese 2 & $\begin{array}{l}\text { Fatores Organizacionais } \\
\text { Linha de Produto } \\
\text { № Total de Funcionários } \\
\text { № de países da operação da } \\
\text { organização } \\
\% \text { funcionários que trabalham } \\
\text { no exterior } \\
\% \text { de vendas de operações } \\
\text { estrangeiras } \\
\text { Local da matriz }\end{array}$ & Escala Completa Global Mindset \\
\hline Hipótese 3 & $\begin{array}{lll}\text { Escala } & \text { Completa } & \text { Global } \\
\text { Mindset } & & \\
\end{array}$ & $\begin{array}{l}\text { Escala Completa Comportamento } \\
\text { Liderança }\end{array}$ \\
\hline
\end{tabular}

Fonte: Konyu-Fogel, 2011. Adaptado pelos pesquisadores

Por fim, a Escala Completa Global Mindset é o escore composto da inteligência intelectual do líder que é composta da habilidade do líder de diferenciar, integrar, gerenciar incerteza, reconhecer padrão e pensar globalmente; e da inteligência cultural que é composta de abertura para aprendizado, sensibilidade emocional, comportamento flexível e entendimento entre culturas (Konyu-Fogel, 2011).

A Escala Completa Comportamento da Liderança é o escore composto das seis dimensões do comportamento da liderança: planejamento, coordenação, liderança, comunicação, motivação e colaboração entre culturas e países para atingir objetivos globais (Konyu-Fogel, 2011).

Para a análise e tratamento dos dados,similar ao método utilizado por Konyu-Fogel (2011).
A ANOVA foi utilizada, inicialmente, para avaliar o efeito das informações organizacionais no global mindset (GM) e, posteriormente, para avaliar o efeito das informações demográficas, também no global mindset.O modelo de regressão linear múltipla foi usado, inicialmente, para avaliar o efeito das informações organizacionais e demográficas, juntas, no global mindset. Nesse momento foram desenvolvidos dois modelos: um modelo completo, sem nenhum método de seleção de variáveis e, posteriormente, foi aplicada a seleção stepwise para chegar em um conjunto de variáveis que expliquem juntas e, de maneira estatisticamente significativa, a relação das informações organizacionais e demográficas com o global mindset.

$\mathrm{O}$ teste qui-quadrado para independência foi utilizado para avaliar, estatisticamente, a associação do 
gênero com a idade, isto é, se havia dependência entre as categorias das duas variáveis em estudo). Devido a independência da distribuição de idade e gênero, estas variáveis foram incluídas nas regressões do estudo como variáveis de controle.

Por fim, a regressão linear múltipla foi usada para avaliar o efeito do global mindset no comportamento da liderança.

Todos os modelos desenvolvidos nesse estudo tiveram as suposições sobre os resíduos satisfeitas, e para tanto foi aplicada a transformação quadrática sobre a variável dependente global mindset, sendo a variável modelada, em todos os modelos desenvolvidos, com o global mindset elevado ao quadrado.

Todos os testes de hipóteses desenvolvidos nesse estudo consideraram 5\% de significância, ou seja, a hipótese nula foi rejeitada quando p-valor foi menor ou igual a 0,05 .

\section{RESULTADOS}

Os participantes reportaram que trabalhavam em organizações onde, dentre as 22 linhas de produto representadas, a mais frequente foi Health Care (44\%), $54 \%$ das organizações possuem mais que 10.000 empregados, $57 \%$ operam entre 51 e 200 países, $47 \%$ possuem mais de $50 \%$ dos empregados no exterior, $55 \%$ possuem mais de $50 \%$ da receita oriunda do exterior e $49 \%$ possuem sede na Europa.

Em relação às informações demográficas, observou-se que $44 \%$ dos participantes possuem entre 47 e 49 anos de idade, $67 \%$ são do sexo masculino, a posição mais frequente foi Diretor(32\%) seguida de Vice-Presidente(14\%), $83 \%$ já trabalhou ou conduziu negócio em mais de 2 países, $88 \%$ falam pelo menos uma língua estrangeira, a minoria(32\%) pertence a família bilíngue/multiétnica, 50\% trabalham na América do Sul, $18 \%$ na Europa e $13 \%$ nos EUA.

Não foi detectada associação estatisticamente significativa, entre idade e gênero, uma vez que p-valor foi igual a 0,1099. O fator organizacional linha de produto e fator demográfico posição possuem associação estatísticamente significativas com o global mindset e apresentaram efeito estatisticamente significativo no global mindset os fatores organizacionais linha de produto igual a $z$ (pvalor=0,0008), Management Consulting(pvalor=0,0326), $\mathrm{R} \& \mathrm{D}(\mathrm{p}$-valor=0,0143), possuuir mais de $50 \%$ da receita oriunda do exterior(pvalor=0,0064),os fatores demográficos posição igual a Recruiting Partner(p-valor=0,0297) e o local de trabalho na América do Sul(p-valor=0,0005).

Em relação às informações demográficas, todos os fatores demográficos foram considerados variáveis independentes em um modelo de ANOVA, avaliando assim, de forma multivariada, o efeito desses fatores na variável dependente global mindset. Como resultado foi detectada associação estatisticamente significativa do local de trabalho com o global mindset, com p-valor igual a 0,0427. A variável família bilíngue é que mais se aproxima da significância, apesar de não tê-la confirmado no teste da ANOVA, já que o p-valor é igual a 0,0572.

Desta forma, como pelo menos um dos fatores demográficos tem efeito significativo no global mindset, a hipótese nula $\mathrm{H} 01$ é rejeitada e a $\mathrm{H} 1$ é aceita, sugerindo que o global mindset dos líderes é significantemente afetado pelo local de trabalho.

Adicionalmente, quando foi efetuada a regressão da variável dependente global mindset sobre as variáveis independentes referentes aos fatores demográficos e organizacionais, seguida da análise de Regressão Stepwise, foi encontrada significância do global mindset com a variável posição e confirmada significância com a variável local do trabalho identificada na ANOVA. Portanto, com base no teste de regressão, o global mindset dos líderes também é significantemente afetado pelo fator demográfico posição.

Em relação às informações organizacionais, todos os fatores organizacionais foram considerados variáveis independentes em um modelo de ANOVA, avaliando assim, de forma multivariada, o efeito desses fatores na variável dependente global mindset. Pelo resultado da ANOVA, em relação às informações organizacionais, não foi detectada associação estatisticamente significativa do global mindset com nenhum dos fatores organizacionais, uma vez que p-valor foi maior que 0,05 para todas as variáveis. Com base no resultado da ANOVA, a hipótese nula $\mathrm{H} 02$ é aceita, sugerindo que o global mindset dos líderes não é significantemente afetado pelos fatores organizacionais.

Quando foi efetuada a regressão da variável dependente global mindset sobre as variáveis independentes referentes aos fatores demográficos e organizacionais, seguida da análise de Regressão Stepwise, foi identificado que as variáveis linha de produto e percentual de receita oriunda do exterior possuem associações estatisticamente significativas com o global mindset, com p-valor menor que 0,05 . Portanto, com o teste de regressão, a hipótese nula $\mathrm{H} 02$ é rejeitada e a H2 é aceita, sugerindo que o global mindset dos líderes é significantemente afetado pelos fatores organizacionais linha de produto e percentual receita oriunda do exterior.

Para avaliar a influencia do global mindset no comportamento da liderança, inicialmente foram calculados os escores de global mindset e comportamento da liderança. Como resultado, obtevese o valor médio para o escore global mindset de 3,87, com mediana igual a 3,91 e desvio padrão de 0,50. Já o escore de comportamento da liderança apresentou valor médio igual a 3,39, com mediana igual a 3,45 e desvio padrão de 0,75 .

Para esta análise foi desenvolvido um modelo de regressão linear múltipla, tendo como variável dependente o escore do comportamento da liderança e 
como variável independente o global mindset. Foi detectado efeito estatisticamente significativo do global mindset no comportamento da liderança, com p-valor menor que 0,0001 e parâmetro estimado positivo de 0,91, sugerindo uma relação positiva entre global mindset e comportamento da liderança.

Portanto, a hipótese nula $\mathrm{H} 03$ foi rejeitada e a H3 foi aceita, indicando que líderes com maior global mindset são mais prováveis de usar comportamentos que demonstrem níveis mais altos nas habilidades de planejamento, coordenação global e forte habilidade para liderar, motivar e colaborar entre culturas e países para atingir atividades globais.

\section{DISCUSSÃO}

Para responder a questão central do estudo, inicialmente foram analisados efeitos fatores demográficos no global mindset e, posteriomente, os efeitos do global mindset no comportamento da liderança.

Em relação à idade, de forma similar às conclusões de Javidan et al.(2009), o estudo mostrou que líderes com idade superior a 60 anos tem maior escore de global mindset(GM = 3,95). Em desacordo com os achados de Knoyu-Fogel(2011) que identificou que os líderes com menor escore global mindset tinham idade entre 26-36 anos e Javidan et al. (2009) que identificou menor escore de global mindset para líderes com idade entre 20-29 anos, o resultado deste estudo mostrou que líderes entre 47-59 anos possuem o menor escore de global mindset $(\mathrm{GM}=3,82)$.

Em relação ao gênero, o estudo mostrou que líderes homens (média $=3,89$ ) possuem o escore global mindset maior que das mulheres(grande média $=3,84$ ). Como a diferença não é significativa, pesquisa futura poderá ser feita com uma amostra maior para confirmar o resultado em comparaçao com as conclusões de Alon e Higgins (2005), Javidan et al.(2006) e Sheridan(2005) de que mulheres são mais prováveis de ter escore global mindset maior que homens.

Em relação ao número de línguas estrangeiras faladas, não foi identificada significância em relação ao escore global mindset, porém o maior escore global mindset foi encontrado para o fator "Três ou mais línguas estrangeiras"(grande média=4,04). Por outro lado, o fator "Nenhuma língua estrangeira" teve o segundo maior escore global mindset. Dos 17 indivíduos que informaram este fator, 94\% trabalham na mesma região que a matriz de suas empresas, o que pode sugerir que estes são indivíduos de países cuja língua nativa é o inglês. Futura pesquisa deve ser realizada para confirmar esta declaração. Pesquisa posterior poderia verificar se com uma amostra maior este resultado será confirmado, já que segundo Laroche et al.(2009), o aprendizado de língua estrangeira está relacionado com o aprendizado da cultura hospedeira e é um portador da identidade individual e coletiva; e segundo Puck et.al. (2008) auxilia no ajuste do expatriado. Adicionalmente, aprender a se comunicar em uma nova língua tem um grande papel na transformação de líderes, como está relacionado a imersão com pessoas e costumes assim como experiências como com a novidade da normalidade (Clapp-Smith e Wernsing, 2014)

Em relação ao fator criado em família bilingue/multiétnica, os resultados do estudo, mostraram que este fator não é significante em relação ao escore global, divergindo de estudos anteriores que destacaram a importância da experiência em ambiente bilingue/multi-etnico e a influência da família no global mindset (Chabowski, 2004) e que destacam que crescer em uma família bicultural pode ser um ativo para uma criança.

Em relação ao número de países onde trabalhou ou conduziu negócios com, o estudo não identificou significância deste fator com o global mindset. Este resultado não confirmou conclusões de estudos anteriores que concluiram que viagens/trabalhos internacionais, imersão cultural intensiva por trabalhar e viver no local, alocações internacionais, e envolvimento em operações em mercados globais e operações globais são prováveis de aumentar o global mindset (Chabowski, 2002; Javidan et.al., 2009 Nummela et.al. 2004 Gregersen et.al. 1998; Leslie et al., 2002; Crowne, 2013) Porém, sugere uma tendência na qual quanto maior o número de países em que trabalhou, maior o global mindset do líder, apesar do maior global mindset estar associado a indivíduos que trabalharam ou conduziram negócios em/com 21 50 países $(\mathrm{GM}=4,13)$ e o segundo maior estar associado a 51-200 países $(\mathrm{GM}=4,10)$.

Em relação ao local de trabalho, o estudo mostrou significância do local de trabalho com o global mindset, que o local de trabalho de $50 \%$ dos participantes é na América do Sul, 18\% na Europa e $13 \%$ nos EUA. Este é um fator significante em relação ao global mindset, conforme o estudo anterior de Konyu-Fogel(2011). Porém, ao contrário do estudo anterior, a maioria da amostra trabalha na America do $\mathrm{Sul}(\mathrm{GM}=3,73)$. Comparando a média das Outras Regiões sem a America do $\operatorname{Sul}(\mathrm{GM}=4,07)$ com a América do Sul, o resultado sugere que os líderes que trabalham fora da América do Sul possuem global mindset maior que os da América do Sul. Da mesma forma, comparando $\mathrm{EUA}(\mathrm{GM}=3,99)$ com Outras Regiões sem os $\operatorname{EUA}(\mathrm{GM}=4,04)$, os líderes de regiões fora dos EUA possuem global mindset maior que os líderes que trabalham nos EUA, confirmando as conclusoes do estudo anterior. Paul et al. (2014) identificaram que a sensibilidade entre culturas é altamente dependente do histórico pessoal do indivíduo, tais como viver fora do país e falar outra língua além do inglês. Ser nativo em inglês, ter crenças políticas conservadoras e morar poucos anos fora dos EUA, foram associados a baixos níveis de sensibilidade entre culturas(Paul et.al., 2011). As diferenças encontradas neste estudo podem estar relacionadas com 
o desenvolvimento de inteligência cultural do indivíduo oriunda de experiências internacionais (Lovvorn e Chen, 2011). Porém, é importante ressaltar que a localização do emprego não representa a nacionalidade do indivíduo. Portanto, conclusões sobre a relação nacionalidade e global mindset podem ser observadas em pesquisas futuras.

Em relação à posição hierarquica, o estudo identificou significância do fator posição com o global mindset. Também apresentou que o maior o escore global mindset foi encontrado para a posição "Recruitment Partner"(GM=4,56), seguida da posição CEO"(GM=4,38), Marketing Executive"(GM=4,15) e Vice Presidente"'(grande média=4,00). Este resultado diverge do estudo anterior, que mostrou as posições Vice Presidente e CEO como as que possuem maior escore global mindset, respectivamente. Por outro lado, o resultado está de acordo com Javidan e Walker(2013) que afirmam que se os profissionais de RH devem estar preparados para liderar esforços de globalização e integração, devem se posicionar para fazer impacto excepcional e verdadeiro para suas organizações, devem desenvolver seu global mindset como uma prioridade.

Em relação ao número total de empregados da empresa, os participantes relataram que $54 \%$ das organizações possuem mais que 10.000 empregados $(\mathrm{GM}=3,93)$, $19 \%$ possuem entre 1.00110.000 empregados $(\mathrm{GM}=3,81), 7 \%$ possuem entre 501-1.000 empregados ( $\mathrm{GM}=3,83)$, e $20 \%$ possuem menos de 500 empregados(GM=3,77). O estudo não apresentou significância deste fator com global mindset, porém sugeriu uma tendênca de que quanto maior o porte da empresa medido pelo número de funcionários maior o global mindset, em oposição à conclusão do estudo anterior(Konyu-Fogel, 2011) que encontrou global mindset maior nas empresas com menos de 500 empregados. O resultado reforça os estudos de Murtha et al.(1998) que concluiram que em corporações multinacionais complexas com mais de 1000 empregados, os líderes provavelmente possuem maior global mindset. O resultado também está de acordo com Jeannet(2000) e Bartlett e Beamish(2014) que concluiram que organizações que empregam um número grande de empregados e são envolvidas em negócios globais provavelmente possuem líderes com maior global mindset, visto que auxiliam a desenvolver estratégias e habilidades globais com capacidade de resposta local.

Em relação a número de países de operação, os participantes relataram que $57 \%$ das empresas operam entre 51 e 200 países $(\mathrm{GM}=3,95), 11 \%$ das empresas operam entre 21 e 50 países $(\mathrm{GM}=3,78), 12 \%$ das empresas operam entre 6 e 20 países $(\mathrm{GM}=3,81)$, $9 \%$ das empresas operam entre 3 e 5 e 200 países $(\mathrm{GM}=3,64)$ e $12 \%$ das empresas operam entre 1 e 2 países $(\mathrm{GM}=3,84)$. O estudo não mostrou significância do número de países de operação da empresa tampouco uma tendência que sugira que quanto maior o número de países da operação maior o global mindset. Este resultado está alinhado com os estudos de Jeannet(2000) que concluiu que embora as organizações operam em vários países, podem não operar com o maior global mindset. Bartlett e Beamish(2014) também afirmam que organizações que operam em diferentes países podem usar estratégias distintas

etnocêntrico(domestico/nacional);policêntrico(diferen ciação multipaís), regiocêntrica(baseada na integração e coordenação regional) ou geogêntrica(global) também chamada de estratégia transnacional. Dependendo da necessidade da operação local e do escopo de atividade, os líderes(domésticos) não necessitam ter global mindset tão alto quanto os dos líderes que tem escopo de atividade global (Javidan e Walker, 2013).

Em relação a linha de produto, este estudo adicionou 6 linhas de produtos à lista do estudo anterior que considerou 14 linhas de produtos. A linha de produto mais frequente foi Health Care (44\%). O estudo anterior encontrou o menor global mindset em Manufatura, Automobilismo e Serviços Profissionais e o maior foi Serviços Bancários e Financeiros. Neste estudo, o menor global mindset foi encontrado em Consulting $(\mathrm{GM}=3,0)$ e o o maior foi Professional Services(GM=4,3). Não foram encontrados estudos que indiquem a relação de linha de produtos com global mindset, portanto novas pesquisas podem aprofundar o estudo sobre esta relação, utilizando amostras maiores.

Em relação ao percentual da receita oriundo do exterior, $55 \%$ dos participantes relataram que suas organizações possuem mais de $50 \%$ da receita gerada no exterior( $\mathrm{GM}=3,99)$, entre de 26 e $50 \%(\mathrm{GM}=3,71)$, de 10 a $25 \%(\mathrm{GM}=3,79)$ e menos de $10 \%(\mathrm{GM}=3,65)$. Foi identificada relação significante entre os escores de global mindset, principalmente entre as organizações cujo é percentual oriundo do exterior é maior(mais de $50 \%$ ), conforme resultado da Regressão Stepwise. Este resultado, confirma as conclusões de e estudos de Bartlett e Beamish (2014): organizações com alta dependência das operações no exterior parecem ter líderes com uma habilidade de integrar e coordenar operações gloabais efetivamente através de vários países e regiões no mundo. Portanto, o resultado sugere que se as organizações aumentam a dependência sobre receita gerada a partir de operações no exterior, líderes parecem ter um global mindset significantemente maior.

Em relação ao local da matriz da organização, $49 \%$ dos participantes relataram que a unidade matriz de suas organizações está localizada na Europa $(\mathrm{GM}=3,94), 25 \%$ nos $\operatorname{EUA}(\mathrm{GM}=3,95), 12 \%$ na América do $\operatorname{Sul}(\mathrm{GM}=3,55), 7 \%$ no Canadá(GM=3,63), $7 \%$ na Ásia $(\mathrm{GM}=3,80)$ e $1 \%$ na África $(\mathrm{GM}=4,75)$. O estudo não demostrou significância do local da matriz da organização com o global mindset, o que sugere que o global mindset dos líderes não é afetado pelo local onde a matriz opera. Por outro lado, o estudo destaca 
que há uma variação significativa entre o global mindset dos líderes das regiões analisadas. Desta forma, recomenda-se estudos mais aprofundados com uma amostra maior.

Por fim, foi encontrado um efeito significante do global mindset no comportamento da liderança: ele é responsável por até $39,1 \%$ de variabilidade no seu comportamento, isto ingifica que identifica-se uma relação direta e positiva entre comportamento da liderança e global mindset, sugerindo que conforme as análises anteriores, é influenciado por fatores demográficos e organizacionais.

Estes resultados confirmam estudos prévios de que líderes globais efetivos usam comportamentos que mostram um entendimento das diferenças entre culturas e países em resposta a ambientes globais e execução de atividades complexas (Rhinesmith, 2003). Em um ambiente global líderes são confrontados com vários desafios e oportunidades, logo são solicitados a se engajarem em planejamento e coordenação complexa, desenvolvimento de habilidades para mobilizarem, motivarem, liderarem e energizarem pessoas, assim como buscarem colaboração e comunicação aberta para atingirem vantagem competitiva sustentável e alta performance(Konyu-Fogel, 2014). Segundo KonyuFogel, as práticas de recursos humanos devem focar em prover suporte para a estratégia da organização e focar no desenvolvimento da liderança que pode ajudar a organização a atingir esta competividade sustentável (Konyu-Fogel, 2013).

Apesar da diversificação da amostra ter gerado uma a lista de fatores demográficos e organizacionais diferente do estudo de Konyu-Fogel (2011), a análise confirmou as hipóteses de que os fatores demográficos e organizacionais afetam o global mindset, bem como o global mindset afeta o comportamento da liderança que é importante para a melhor performance das organizações. Identificamos na pesquisa que líderes que trabalham na America do Sul apresentaram global mindset menor que a média das demais regiões e recomendamos para a prática gerencial direcionar ações específicas para que a liderança atue com maiores níveis de global mindset, e, programas de desenvolvimento de liderança, medindo o global mindset de todos os níveis de sua cadeia gerencial que atuem em atividades com responsabilidade global; e para o campo acadêmico, futuras pesquisas, que incluíam uma análise qualitativa para aprofundar conclusões e comparações com o trabalho de KonyuFogel(2014).

$\mathrm{Na}$ tabela 5 resumimos os principais resultados das análises que respondem as questões de pesquisa desta pesquisa:

Tabela 5: Questões da pesquisa e resultados encontrados

\begin{tabular}{|c|c|}
\hline QUESTOES & RESULTADOS \\
\hline $\begin{array}{l}\text { Os fatores demográficos } \\
\text { influenciam o global mindset } \\
\text { dos líderes de negócios? }\end{array}$ & $\begin{array}{l}\text { Idade, gênero, número de línguas estrangeiras, criação em família } \\
\text { bilingue/multiétnica, número de países onde trabalhou ou conduziu negócios } \\
\text { não afetam o global mindset, enquanto os fatores demográficos, posição e local } \\
\text { de trabalho afetam o global mindset dos líderes de negócio. }\end{array}$ \\
\hline $\begin{array}{l}\text { Os fatores organizacionais } \\
\text { influenciam o global mindset } \\
\text { dos líderes de negócios? }\end{array}$ & $\begin{array}{l}\text { O número total de empregados, número de países que a empresa opera, } \\
\text { percentual de empregados no exterior e local da matriz não afetam } \\
\text { significantemente o global mindset enquanto, percentual de receita oriunda do } \\
\text { exterior e linha de produto o impactam com significância. }\end{array}$ \\
\hline $\begin{array}{l}\text { O global mindset dos líderes } \\
\text { de negócio afeta o } \\
\text { comportamento da liderança } \\
\text { que é critico para o } \\
\text { desempenho organizacional? }\end{array}$ & $\begin{array}{l}\text { Foi encontrado um efeito significante do global mindset no comportamento da } \\
\text { liderança, indicando que o global mindset é responsável por até } 39,1 \% \text { de } \\
\text { variabilidade no comportamento da liderança. Este resultado implica em uma } \\
\text { relação direta e positiva entre comportamento da liderança e global mindset, } \\
\text { sugerindo que o comportamento da liderança é esperado variar } \\
\text { significativamente baseado no global mindset, que conforme as análises } \\
\text { anteriores, é influenciado por fatores demográficos e organizacionais. }\end{array}$ \\
\hline $\begin{array}{l}\text { Quais os fatores que afetam o } \\
\text { global mindset de líderes } \\
\text { globais e qual o seu efeito no } \\
\text { seu comportamento? }\end{array}$ & $\begin{array}{l}\text { Estudo confirmou que os fatores demográficos e organizacionais influenciam } \\
\text { o global mindset dos líderes, reduzindo ou aumentando o global mindset, que } \\
\text { influencia o comportamento da liderança. } \\
\text { O comportamento da liderança que é crítico para a organização é provável de } \\
\text { aumentar se o global mindset aumenta. }\end{array}$ \\
\hline
\end{tabular}

Fonte: Elaborado pelos autores 


\section{CONCLUSOES}

As organizações parecem não estar preparadas o suficiente para preparar líderes para o ambiente de negócios internacional (Mendenhall et al., 2013). É importante atentar que a definição de líder global não está restrita ao alto executivo da empresa; qualquer pessoa que lidere esforços de mudança global no setores público, privado multinacional ou nacional e não lucrativo é um global líder (Javidan e Walker., 2013; Mendenhall et al., 2013).

Este estudo contribui em trazer reflexões sobre o efeito do global mindset no comportamento da liderança, não somente sob o ponto do alto executivo da empresa, mas também de líderes que não estão na primeira linha executiva da empresa, porém atuam em atividades com interações ou responsabilidades globais. Portanto, este estudo reforça posição de estudos anteriores de que o desenvolvimento dos líderes deve atingir todos os níveis da organização que interajam em esfera global (Jeannet, 2000).

O global mindset foi referenciado por vários autores neste estudo como característica chave para o desenvolvimento da liderança e, de forma específica, este estudo confirmou que o global mindset afeta o comportamento da liderança. Reforçando as definições da literatura revisada neste estudo, recomenda-se que organizações que atuem em ambiente global e que necessitem manter ou aumentar vantagem competitiva em seu mercado, reforcem seus programas de desenvolvimento de liderança, medindo o global mindset de todos os níveis de sua cadeia gerencial que atuem em atividades com interface ou responsabilidade global. Como resultado, será possível direcionar ações específicas para obter planos de desenvolvimento para que a liderança atue mais estratégicamente.

Em geral não é quantificado o quanto se desperdiça de tempo em tomada de decisões ou até mesmo em direcionamentos locais ou globais ineficazes para a organização, decorrentes de visão limitada com baixo ou inexistente global mindset da liderança. As politicas gerenciais em geral e as de recursos humanos em particular, devem prover suporte para a estratégia da organização e focar no desenvolvimento da liderança para auxiliar a organização a se tornar mais competiva através de habilidades e competências chave sustentáveis (KonyuFogel, 2013).

\section{REFERÊNCIAS}

Adler, N. J.; Bartholomew, S.(1992) Managing globally competent people. Academy of Management Executive, 6(3), 52.

Aggarwal, R. (2011) Developing a global mindset: Integrating demographics, sustainability, technology, and globalization. Journal of Teaching in International Business, v. 22, n. 1, p. 51-69.

Alon, I.; Higgins, J. M. (2005) Global leadership success through emotional and cultural intelligences. Business horizons, v. 48, n. 6, p. 501-512.

Ananthram, S.; Pearson, C.; Chatterjee, S. (2010) Do organizational reform measures impact on global mindset intensity of managers? Journal of Chinese Economic and Foreign Trade Studies, v. 3, n. 2, p. 146168.

Arora, A.; Jaju, A.; Kefalas, A. G.; Perenich, T. (2004) An exploratory analysis of global managerial mindsets: a case of U.S. textile and apparel industry. v. 10, n. 3, p. 393-411.

Bartlett, C. A.; Beamish, P. W. (2014)

Transnational Management: text, cases, and reading in cross-border management. Seventh edition. Singapore.

Beechler, S.; Javidan, M. (2007) Leading with a global mindset. Advances in international management, v. 19, p. 131-169.

Black, J. S.; Gregersen, H. B. (2000) High impact training: Forging leaders for the global frontier. Human Resource Management, v. 39, n. 2-3, p. 173-184.

Black, K. (2012) Business Statistics For Contemporary Decision Making. $7^{\text {th }}$ Edition. US. John Wiley \& Sons, Inc.

Blaess, D. A.; Hollywood, K. G.; Grant, C. (2012) Preparing the professoriate to prepare globally competent leaders. Journal of Leadership Studies, v. 6, n. 2, p. 88-94.

Bouquet, C.; Morrison, A.; Birkinshaw, J. (2009) International Attention and Multinational Enterprise Performance. Journal of International Business Studies, v. 40, n. 1, p. 108-131.

Bowen, D. E., Inkpen, A. C. (2009) Exploring the Role of "Global Mindset" in Leading Change in International Contexts. The Journal of Applied Behavioral Science, v. 45, n. 2, p. 239-260. 
Brake, T. (1997) The global leader: Critical factors for creating the world class organization. Chicago, IL: Irwing Professional Publishing.

Calof, J.; Beamish, P. (1994).The right attitude for international success. Business Quartely. v. 59(1), p. 105-110.

Calori, R., Johnson, G., \& Sarmin, P. (1994) $C E O$ 's cognitive maps and the scope of the organization. Strategic Management Journal, 15 (6), 437-457.

ChabowskI, B. R. (2002)Developing global executives: the lessons of international experience: Morgan W. McCall, Jr. and George P. Hollenbeck, Harvard Business School Press, 259 pp., v. 13, n. 3, p. 419-422.

Clapp-Smith, R.; Wernsing, T. (2014) The transformational triggers of international experiences. Journal of Management Development, v. 33, n. 7, p. 662-679.

Cohen, S. L. (2010) Effective global leadership requires a global mindset. Industrial and Commercial Training, v. 42, n. 1, p. 3-10.

Crowne, K. A. (2013) Cultural exposure, emotional intelligence, and cultural intelligence: An exploratory study. International Journal of Cross Cultural Management, v. 13, n. 1, p. 5-22, April.

Dorfman, P.; Javidan, M.; Hanges, P.; Dastmalchian, A.; House, R. (2012) GLOBE: A twenty-year journey into the intriguing world of culture and leadership. Journal of World Business, v. 47, n. 4, p. 504-518, October.

Dreher, A. (2006) Does globalization affect growth. Evidence from a new index of globalization. Applied Economics, v. 38, n. 10, p. 1091-1110.

Earley, P. C.; Mosakowski, E. (2004) Cultural Intelligence. Harvard Business Review, v. 82, n. 10, p. 139-146.

Fatehi, K., Ghadar, F. (2014) A measure of international managers' mindset. International Journal of Commerce and Management, v. 24, n. 4, p. 321-333.

Gabel, M.; Bruner, H. (2003) Global Inc.: an Atlas of the Multinational Corporation. New York, NY.

Goldsmith, M.; Greenberg, C. L.; Roberston, A.; Hu-Chan, M. (2003). Global Leadership, The Next Generation. HALL, P. NJ.
Gregersen, H. B.; Morrison, A. J.; Black, J. S. (1998) Developing leaders for the global frontier. Sloan Management Review, v. 40, n. 1, p. 21-32.

Gundling, E.; Hogan, T.; Cvitkovich, K. (2011) What is Global Leadership. Nicholas Brealey. US.

Gupta, A. K.; Govindarajan, V. (2002) Cultivating a global mindset. Academy of Management Executive, v. 16, n. 1, p. 116-126.

Gupta, A. K., Wang, H. (2008). Global enterprise 2020. Chief Executive, 234, 32-36.

Heenan, D. A.; Perlmutter, H. V. (1979)

Multinational organizational development: A social architecture perspective. MA: Addison-Wesley.

House, R.; Javidan, M.; Hanges, P.; Dorfman, P. (2002) Understanding cultures and implicit leadership theories across the globe: an introduction to project GLOBE. Journal of World Business, v. 37, n. 1, p. 310 .

House, R.; Javidan, M.; Dorfman, P. (2001) Project GLOBE: an introduction. Applied Psychology, v. 50, n. 4, p. 489-505.

Javidan, M.; Bowen, D. (2013) 'Global Mindset' of managers. Organizational dynamics, v. 42, n. 2, p. 145.

. (2013) The 'Global Mindset' of managers: What it is, why it matters, and how to develop it. Organizational Dynamics, v. 42, n. 2, p. 145-155.

Javidan, M.; House, R.; Dorfman P.; Hanges, P; Luque, M. S. (2006). Conceptualizing and measuring cultures and their consequences: a comparative review of GLOBE's and Hofstede's approaches. Journal of international business studies, v. 37, n. 6, p. 897-914.

Javidan, M.; Hough, L.; Bullough, A. (2009) Conceptualizing and Measuring Global Mindset: Development of the Global Mindset Inventory. Glendale, AZ.

Javidan, M.; Walker L., J. (2013) Developing Your Global Mindset: The Handbook for Successful Global Leaders eBook: Mansour Javidan, Jennie L Walker [Kindle Edition]. Edina, MN: Beaver's Pond Press, Inc.

Jeannet, J. (2000) Managing with a global mindset. London: Pearson Education.

Kedia, B. L.; Mukherji, A. (1999) Global managers: developing a mindset for global competitiveness. Journal of World Business, v. 34, n. 3, p. 230-251. 
Kefalas, A. G. (1998) Think globally, act locally. Thunderbird International Business Review, v. 40, n. 6, p. 547-562.

Kobrin, S. J. (1994) Is There a Relationship between a Geocentric Mindset and Multinational Strategy, v. 25, n. 3, p. 493-511.

Konyu-Fogel, G. (2011) Exploring the effect of global mindset on leadership behavior: An empirical study of business leaders in global organizations. ProQuest Dissertations and Theses, Lawrence Technological University, Ann Arbor.

(2013). Global Skills and Competency Training for Developing Global Talent: Implications for International Human Resource Management. Journal of the North American Management Society, v. 7, n. 1, p. 13.

(2014) 21st Century Sustainable Competitive Advantage: Critical Capabilities through Leadership and Organizational Development. Economics and Culture Scientific Journal, Riga, Latvia, v. Vol. 10 (1), p. 29-46.

Lane, H. W.; Maznevski, M.; Mendenhall, M. E.; McNett, J. (2004) The Blackwell handbook of global management: A guide to managing complexity. UK: Blackwell Publishing.

Laroche, M.; Pons, F.; Richard, M. (2009) The Role of Language in Ethnic Identity Measurement: A Multitrait-Multimethod Approach to Construct. The Journal of Social Psychology, 2009, 149(4), 513-539. Heldref Publications.

Leslie, J. B.; Dalton, M.; Ernst, C.; Deal, J. (2002) Managerial Effectiveness in a Global Context. North Carolin: Center for Creative Leadership.

Levy, O.; Beechler, S.; Taylor, S.; Boyacigiller, N. A. (2007) What We Talk about When We Talk about 'Global Mindset': Managerial Cognition in Multinational Corporations. Journal of International Business Studies, v. 38, n. 2, p. 231-258.

Liedtka, J. |(1991) Organizational value contention and managerial mindsets. Journal of Business Ethics, v. 10, n. 7 , p. $543-557$.

Lovvorn, A. S.; Chen, J.-S. (2011) Developing a global mindset: The relationship between an international assignment and cultural intelligence. International Journal of Business and Social Science, v. 2, n. 9 , p. 275-283.

Mendenhall, M. E.; Osland, J. S.; Bird, A. Oddou, G. R.; Maznevski M. L.; Stevens, M.J.; Stahl, G. K.
(2013) Global Leadership: Research, Practice and Development. New York: Taylor \& Francis.

Mendenhall, M. E.; Reich, B. S; Osland, J. S.; Bird, A. (2012) Defining the "global" in global leadership. Journal of World Business, v. 47, n. 4, p. 493-503, October.

Mendenhall, M. E.; Osland, J. S.; Bird, A. Oddou, G. R.; Stevens, M.J. (2008) Specification of the content domain of the Global Competencies Inventory (GCI). The Kozai Working Papers Series.

Miriam, E.; Lisak, A.; Harush, R.; Glikson E.; Nouri, R.; Shokef, E. (2013). Going Global: Developing Management Students' Cultural Intelligence and Global Identity in Culturally Diverse Virtual Teams. Academy of Management Learning \& Education, v. 12, n. 3, p. 330-355.

Morley, M. J.; Cerdin, J. L. (2010). Global management competencies: a theoretical foundation. Journal of Managerial Psychology, v. 25, n. 8, p. 829844.

Morrison, A. J. (2000) Developing a global leadership model. Human Resource Management, v. 39 , n. 2-3, p. 117-131.

Maak T., Pless, N. M. (2009) Business Leaders as citizens of the world: Advancing humanism on a global scale. Journal of Business Ethics, 88, 537-550.

Murtha, T., Lenway, S. A., Bagozzi, R. P. (1998) Global mindsets and cognitive shift in complex multinational corporations. Strategic Management Journal, 19, 97-114.

Nummela, N.; Saarenketo, S.; Puumalainen, K. (2004) A Global Mindset :A Prerequisite for Successful Internationalization? Canadian Journal of Administrative Sciences, v. 21, n. 1, p. 51-64.

Oddou, G.; Mendenhall, M. E.; Ritchie, J. B. (2000) Leveraging travel as a tool for global leadership development. Human Resource Management, v. 39, n. 23 , p. 159-172.

Paul, H. (2000) Creating a global mindset. Thunderbird International Business Review, v. 42, n. 2 , p. 187-200.

Paul, K.; Meyskens, M.; Robbins, S. (2011) Components of a global mindset: corporate social responsibility and cross-cultural sensitivity. Journal of International Business and Cultural Studies, v. 5, n. 1. 
Perlmutter, H. V. (1969) The Tortuous Evolution of the Multinational Corporation. Columbia Journal of World Business, v. 4, n. 1, p. 9.

Prahalad, C. K.; Doz, Y. (1987) The multinational mission: Balancing local demands and global vision. New York, NY: Free Press.

PriceWaterhouseCoopers. PWC 17th Annual Global CEO Survey. The Desing Group PwC. UK. 2014.

Pucik, V.; Saba, T. (1998). Selecting and developing the global versus the expatriate manager: a review of the state-of-the-art. 21: $40 \mathrm{p}$.

Puck, J. F.; Kittler, M. G.; Wright, C. (2008) Does it really work? International journal of human resource management, v. 19, n. 12, p. 2182-2197.

Punnet, B. J.; Shenkar, O.(2004) Handbook for International Management Research. 2nd ed. Michigan, US: Michigan University.

Ranker, G.; Huang, D.; Mcleod, M. (2015) Navigating US and Chinese business cultures using global mindset leadership. Training \& Development $\mathrm{v}$. 2015 , n. 75 , p. $23-30$.

Rhinesmith, S. H. (1996) A manager's guide to globalization: six steps to success in a changing world. New York, NY: Irwing Publishing.

(2003) Basic components of a global

mindset. In Goldsmith, M., Govidarajan, B., \& Vicere, A. (Eds.) The many facets of leadership. Upper Saddler River, NJ: Financial Times Prentice Hall.

Robinson, D. A.; Harvey, M. (2008) Global leadership in a culturally diverse world. Management Decision, v. 46, n. 3, p. 466-488.

Rogers, E. M.; Blonski, D. (2010) The global leadership mindset. Chief Learning Officer, June, p. 18 21.
Sambharya, R. (1996) Foreign experience of top management teams and international diversification strategies of U.S. multinational corporations. Strategic Management Journal, 17, 739-746.

Sheridan, E. (2005) Intercultural leadership competencies for United States business leaders in the new millennium. ProQuest Dissertations and Theses, University of Phoenix, Ann Arbor.

Story, J. S. P.; Barbuto, J. E; Luthans, F.; Bavaird, J. A. (2014). Meeting the Challenges of Effective International HRM: Analysis of the Antecedents of Global Mindset. Human Resource Management, v. 53, n. 1, p. 131-155.

Story, J. S. P.; Barbuto, J. E. (2011) Global Mindset: A Construct Clarification and Framework. Journal of Leadership \& Organizational Studies, v. 18, n. 3, p. 377-384.

Taylor, S.; Levy, O.; Boyacigiller, N. A; Beechler, S. (2008). Employee commitment in MNCs: Impacts of organizational culture, HRM and top management orientations. International Journal of Human Resource Management, v. 19, n. 4, p. 501-527.

Triandis, H. C. (2006) Cultural Intelligence in Organizations. Group \& Organization Management, v. 31, n. 1, p. $20-26$.

Trompenaars, f.; Hampden-Turner, C.(1998) Ridsing the waves of culture. 2nd ed. New York, NY: McGraw-Hill, 1998.

Tubbs, S. L.; Schulz, E. (2006) Exploring a Taxonomy of Global Leadership Competencies and Meta-competencies. The journal of American Academy of Business, Cambridge, v. 8, n. 2, p. 29.

Unit, E. I.(2010) "Global firms in 2020: The next decade of change for organizations and workers." Retrieved January, 20, 2015.

World Economic Forum - Global Agenda Outlook (2015). Switzerland. 